International Journal of Case Reports
(ISSN:2572-8776)

\title{
Oral pyridostigmine for treatment of postoperative ileus associated with elevated catecholamine levels: A case report
}

\author{
Sherif Aly, MD ${ }^{1,2}$ and Gentian Kristo, MD, MPH, FACS ${ }^{1,3 .}$
}

${ }^{1}$ Department of Surgery, Veterans Affairs Boston Healthcare System, Boston, MA, USA; ${ }^{2}$ Department of Surgery, Boston Medical Center, Boston University School of Medicine, Boston, MA, USA; ' ${ }^{3}$ Department of Surgery, Brigham and Women's Hospital, Harvard Medical School, Boston, MA, USA.

\section{ABSTRACT}

The etiology of post-operative ileus is usually multifactorial, but increased catecholamines have been implicated as an important cause. We present a case of catecholamine-induced prolonged post-operative ileus treated successfully with Pyridostigmine. A 70-year-old male underwent a low-anterior resection and diverting loop ileostomy for rectal cancer. Immediately post-operatively he developed refractory hypertensive urgency and a small bowel ileus. Biochemical testing revealed markedly elevated 24-hour urinary metanephrines and normetanephrines. However, radiologic studies failed to identify a pheochromocytoma. The ileus persisted despite employing a multimodal regimen consisting of avoidance of narcotic pain medications, gastric decompression via a nasogastric tube, maintenance of normal levels of electrolytes, parenteral nutritional support, and early mobilization. Two weeks after the surgery the patient was treated with oral Pyridostigmine with appropriate return of bowel function. Excessive circulating catecholamines play an important role in the etiology of refractory post-operative ileus, and cholinesterase inhibitors such as Pyridostigmine could be used as an effective treatment in such cases.

Keywords: post-operative ileus; catecholamines; pheochromocytoma; cholinesterase inhibitor

Disclosure Statement: The authors have no conflicts of interest or financial disclosures to declare.
*Correspondence to Author: Gentian Kristo, MD, MPH, FACS Department of Surgery, Veterans Affairs Boston Healthcare System (116-C) 1400 VFW Parkway, West Roxbury, MA, 02132, USA

How to cite this article:

Sherif Aly and Gentian Kristo. Oral pyridostigmine for treatment of postoperative ileus associated with elevated catecholamine levels: A case report. International Journal of Case Reports, 2019 4:77

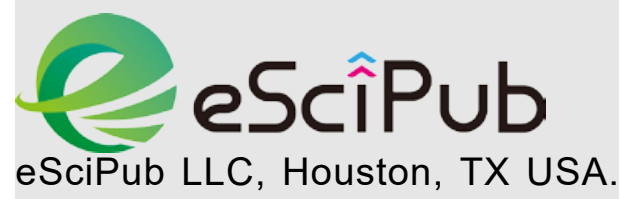

Website: http://escipub.com/ 


\section{Introduction}

Prolonged ( $>3-5$ days) postoperative ileus can increase patient discomfort, prevent adequate nutrition, prolong hospitalization, and increase healthcare expenses ${ }^{1}$. The etiology of the postoperative ileus is multifactorial, with increased catecholamines being an important cause. Elevated catecholamine levels after abdominal surgery are known to reduce the gastrointestinal peristaltic activity ${ }^{2}$ and can result in an ileus. In this report we present our experience with a case of refractory postoperative ileus that occurred in the context of an excessive catecholamine release.

\section{Case Report}

A 70-year-old man with chronic left hydronephrosis and rectal cancer underwent a low-anterior resection and a diverting loop ileostomy. A full mobilization of the splenic flexure of the colon was performed in order to achieve adequate colonic redundancy to allow for a tension-free colorectal anastomosis. Two hours after the surgery the patient developed significant abdominal distention and hypertensive urgency (with systolic blood pressures ranging from $160-230 \mathrm{~mm} \mathrm{Hg}$ ). A nasogastric tube was placed for gastric decompression and a subsequent abdominal $X$ ray showed uniform dilatation of the small bowel loops suggestive of an immediate post-operative ileus (Figure 1).

With hypertension refractory to intermittent intravenous doses of different antihypertensive medications, the patient was started on continuous infusions of nitrates (Nitroglycerin) and calcium channel blockers (Nicardipine), which he required for a total of 5 days.

Given persistence of the ileus and the inability to tolerate oral intake, the patient was started on total parenteral nutrition on post-operative day (POD) seven. A computed tomography (CT) scan of the abdomen obtained on POD 12 ruled out a post-operative intestinal obstruction (Figure 2).
The immediate and prolonged postoperative hypertensive urgency and ileus indicated a significant catecholamine surge and raised concerns of an underlying, undiagnosed pheochromocytoma. Biochemical tests performed on POD 14 showed markedly elevated 24-hour urinary metanephrines [680 $\mathrm{mcg}(90-315)]$ and normetanephrines [1229 mcg (122-676)], as well as significantly increased plasma chromogranin A levels [2668 ng/ml $(25-$ 140). No pheochromocytoma was identified on a subsequent radiologic work-up that included CT scan of the abdomen and pelvis; retroperitoneal ultrasound; and Gallium 68 (68-Ga) 1,4,7,10tetraazacyclododecane-1,4,7,10 - tetraacetic acid (DOTA)-octreotate (DOTATATE)-positron emission tomography (68-Ga DOTATATE PET).

In the absence of evidence suggesting a pheochromocytoma diagnosis, the increased levels of 24-hour urinary fractionated metanephrines and catecholamines were attributed to an abnormally elevated catecholamine release in response to surgical stress. The high plasma chromogranin A levels were considered a non-specific marker of the patient's rectal cancer.

After POD 5, we were able to control the patient's hypertension with intermittent intravenous doses of various antihypertensive medications. However, the small bowel ileus persisted on POD 14 despite employing a multimodal regimen consisting of avoidance of narcotic pain medications; gastric decompression via a nasogastric tube; maintenance of normal levels of electrolytes; parenteral nutritional support; and early mobilization. As such, the patient was started on the reversible cholinesterase inhibitor, Pyridostigmine, $15 \mathrm{mg}$ orally, every 8 hours. The nasogastric tube was clamped for 1 hour after each dose of Pyridostigmine to allow for its absorption. After receiving a total of 3 doses of Pyridostigmine, the patient started passing flatus and having return of bowel function through his ileostomy, marking the resolution of a prolonged post-operative ileus. The Pyridostigmine was 
Sherif Aly and Gentian Kristo, IJCR, 2019 4:77

discontinued and the patient was gradually Repeat biochemical tests performed as an started on oral nutrition. The remainder of the outpatient on POD 43 showed normalized 24post-operative course was uneventful, and hour urinary metanephrines [79 mcg (90-315)] subsequently the patient was discharged home and normetanephrines [207 mcg (122-676)], as and had an unremarkable recovery. well as normal levels of urinary 5hydroxyindoleacetic acid (5-HIAA).

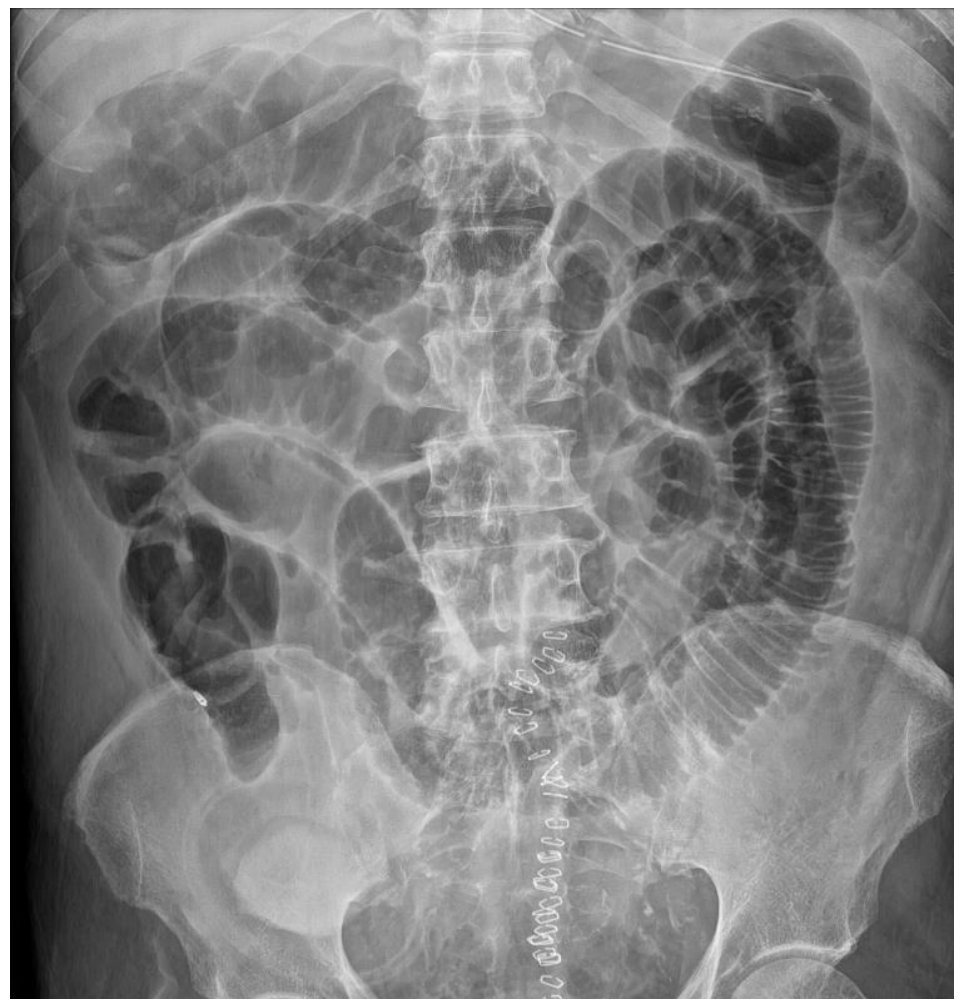

Figure 1. Abdominal X-ray showing dilated loops of small bowel

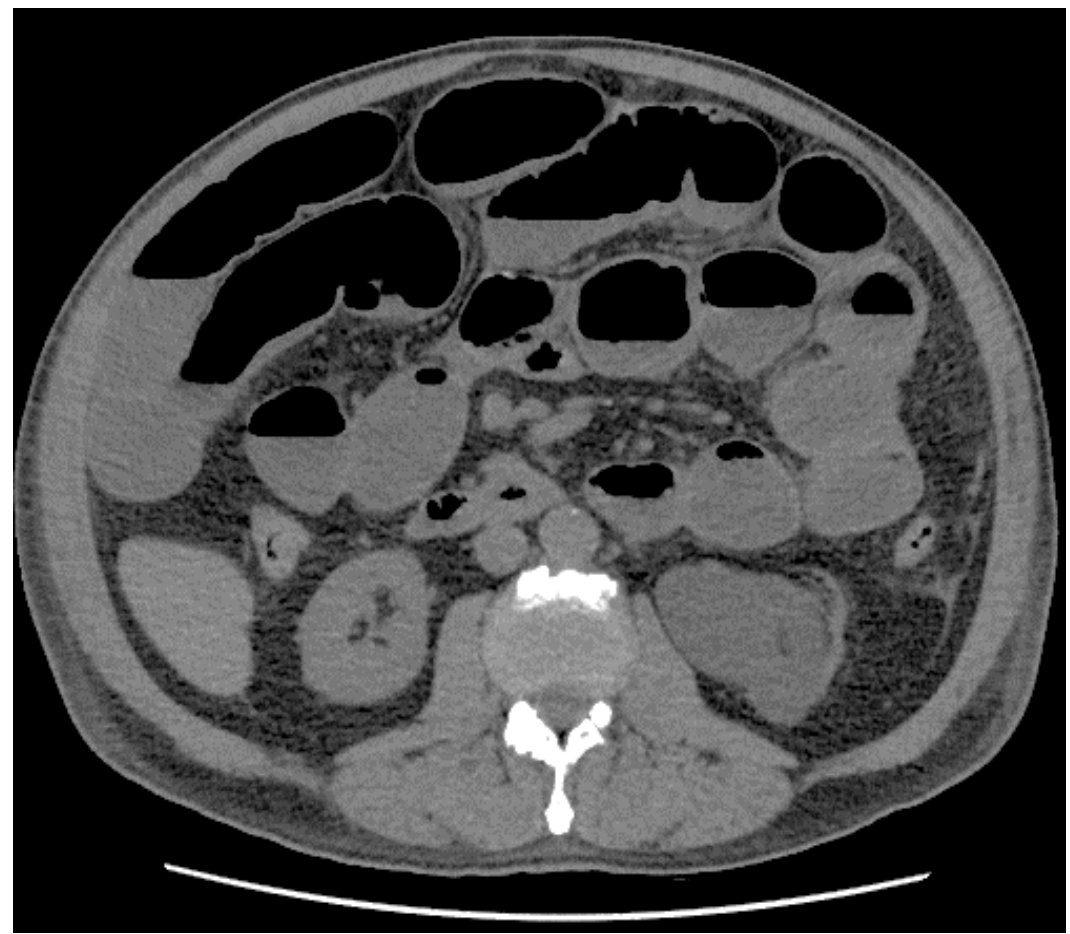

Figure 2. Axial CT scan of the abdomen demonstrating dilated loops of small bowel without a transition point to suggest a mechanical bowel obstruction 


\section{Discussion}

Herein, we report the case of a patient with an immediate-onset and prolonged postoperative ileus caused by an intense catecholamine release and resolved by the administration of Pyridostigmine, a reversible cholinesterase inhibitor.

Paralytic postoperative ileus is a significant clinical problem with a multifactorial etiology that includes inflammation, effects of opioids, release of neurotransmitters and hormones, and inhibitory sympathetic activity ${ }^{2}$. The regulation of bowel motility is balanced between excitatory and inhibitory input from the autonomic nervous system. The parasympathetic nerve endings release acetylcholine, which increases gastrointestinal motility through activation of muscarinic receptors ${ }^{3}$. In contrast, the sympathetic nerve endings release catecholamines, which not only decrease gastro-intestinal motility by activating adrenergic receptors ${ }^{4}$, but also cause a presynaptic catecholamine-mediated inhibition of parasympathetic acetylcholine release ${ }^{3,5}$.

The relationship between high catecholamine levels and reduced gastrointestinal motility is evident in patients with pheochromocytomas, which are catecholamine-secreting tumors. There are several case reports of pheochromocytomas that manifested as paralytic ileus ${ }^{6-8}$, constipation ${ }^{8,9}$, or pseudoobstruction ${ }^{10-12}$.

Our patient developed an immediate postoperative release of excess catecholamines in his circulation which caused a refractory hypertensive urgency and inhibited the gastrointestinal motility resulting in a refractory, paralytic ileus. While catecholamines are normally secreted during surgery, the postoperative catecholamine response in our patient was unusually strong. Although the cause of this exacerbated response remains elusive, it is possible that inadvertent manual stimulation of the left hydronephrotic kidney and/or left suprarenal gland during mobilization of the splenic flexure of the colon could have caused hypersecretion of catecholamines.

Pyridostigmine is a reversible cholinesterase inhibitor that prevents the degradation of acetylcholine and increases its presynaptic concentration ${ }^{13}$. As such, Pyridostigmine has the potential to counteract the presynaptic catecholamine-mediated inhibition of parasympathetic acetylcholine release. By increasing acetylcholine, the gastrointestinal motility is augmented.

Thus, pyridostigmine is shown to reduce constipation in patients with Parkinson's disease and autoimmune neuropathy ${ }^{14}$, as well as in other patients with refractory chronic constipation $^{15}$. Furthermore, one study has shown that oral pyridostigmine is an effective and safe treatment for postoperative ileus ${ }^{16}$.

The dose of pyridostigmine that we administered was well tolerated by our patient, without any cholinergic or cardiovascular side effects. However, the use of reversible cholinesterase inhibitor drugs such as pyridostigmine can lead to severe bradycardia in patients with bradyarrhythmias or in those receiving betaadrenergic antagonists. Therefore, close monitoring for possible side effects is recommended.

\section{Conclusions}

Our report indicates that cholinesterase inhibitors such as Pyridostigmine are effective in the treatment of refractory paralytic ileus in the presence of excessive circulating catecholamine levels. Oral pyridostigmine could be used as an adjunctive treatment in patients with prolonged postoperative ileus.

\section{References}

1. Asgeirsson $\mathrm{T}$, El-Badawi $\mathrm{KI}$, Luchtefeld $\mathrm{M}$, Senagore AJ, et al. Postoperative ileus: It costs more than you expect. J. Am. Coll. Surg. 2010, 210, 228-231.

2. Luckey A, Livingston E, Taché Y. Mechanisms and Treatment of Postoperative lleus. Arch Surg. 
2003;138(2):206-214.

doi:10.1001/archsurg.138.2.206.

3. Wood JD. Intrinsic neural control of intestinal motility. Annu Rev Physiol. 1981;43:33-51.

4. Wood JD. Neurotransmission at the interface of sympathetic and enteric divisions of the autonomic nervous system. Chin J Physiol. 1999;42:201-10.

5. Wood JD, Mayer CJ. Adrenergic inhibition of serotonin release from neurons in guinea pig Auerback's plexus. J Neurophysiol. 1979;42:594603.

6. R. Cruz S, Colwell J. Pheochromocytoma and Ileus. JAMA. 1972. 219. 1050-1051.

7. Noguchi M, Taniya T, Ueno K, Miyazaki I, et al. A case of pheochromocytoma with severe paralytic ileus. Jpn J Surg. 1990 Jul;20(4):448-52.

8. Seung-Joon $\mathrm{H}$, Mi-kwang $\mathrm{K}$, Suk $\mathrm{Ch}$. A case of retroperitoneal paraganglioma manifested as intractable constipation with paralytic ileus and aggravated hyperglycemia. J Korean Endocr Soc. 2008 Dec;23(6):450-455.

9. Thosani S, Ayala-Ramirez M, Roman-Gonzalez A, Jimenez C, et al. Constipation: an overlooked, unmanaged symptom of patients with pheochromocytoma and sympathetic paraganglioma. European Journal of Endocrinology. 2015; 173:377-387.
10. Turner CE. Gastrointestinal pseudo-obstruction due to pheochromocytoma. Am J Gastroenterol. 1983 Apr;78(4):214-7.

11. Sweeney AT, Malabanan AO, Blake AM, Melby $\mathrm{JC}$, et al. Megacolon as the Presenting Feature in Pheochromocytoma. The Journal of Clinical Endocrinology \& Metabolism. 2000 Nov;85(11):3968-3972.

12. de Lloyd AC, Munigoti S, Davies JS, ScottCoombes D. A rare and life-threatening cause of pseudo-obstruction in two surgical patients. BMJ Case Reports 2010.

13. Malekzadeh F, Ansari R, Malekzadeh R. Do you mean: Approach to chronic constipation. Govaresh 2014; 19: 7-13.

14. Sadjadpour K. Pyridostigmine bromide and constipation in Parkinson's disease. JAMA 1983; 249: 1148-60.

15. Soufi-Afshar I, Moghadamnia A, Bijani A, ShokriShirvani $\mathrm{J}$, et al. Comparison of pyridostigmine and bisacodyl in the treatment of refractory chronic constipation. Caspian J Intern Med 2016; 7(1):1924.

16. Maleknejad A, Khazaei A, Bouya S. Evaluation of the effect of oral pyridostigmine on the ileus after abdominal surgery: a blinded randomized clinical trial. J. Clin. Med. 2018, 7, 104.

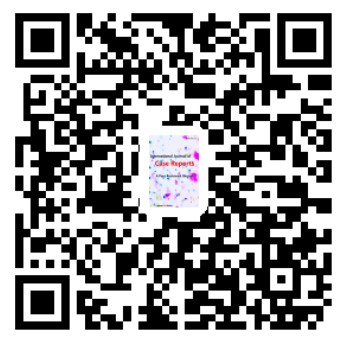

